\section{Let there be light}

bioRxiv http://doi.org/ggbdcj (2019).

bioRxiv http://doi.org/dc5x (2019).

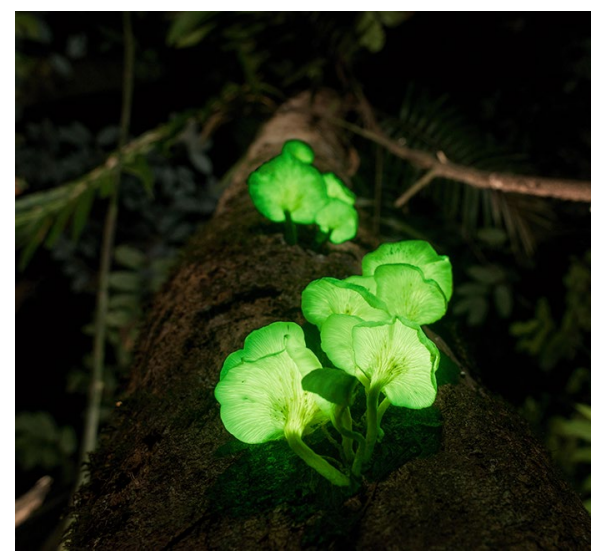

Credit: Nature Picture Library / Alamy Stock Photo

Bioluminescent plants have always existed - but only in the imagination of science fiction enthusiasts. In the real world, various species of insects, fungi, bacteria, marine animals, dinoflagellates and so on can produce light on their own, but no known plant displays autogenic bioluminescence. Scientists have been trying to correct this unfortunate evolutionary fact for some time. Autonomously glowing plants, that do not need the addition of exogenous substrate with unpredictable diffusion, have been designed before. These rare bioengineering advances, often clouded by exaggerated marketing claims, silly headlines and unrealistic promises to replace electric light bulbs, started almost a decade ago with transplastomic tobacco transformed with a full bacterial lux operon.

Now, in two preprints simultaneously published on bioRxiv, two groups propose a new and promising approach based on a recently discovered fungal luminescence biochemical pathway. Three genes are enough to produce and recycle luciferin from caffeic acid, a ubiquitous plant metabolite central in the phenylpropanoid pathway. A fourth fungal gene encodes the luciferase that will oxidize luciferin and produce visible photons. The amount of light, an order of magnitude higher than in previous efforts, is enough to see the glow with naked eyes, or record it with only a few seconds of exposure time with nonspecialized cameras. The emitted light is green, which minimizes interference from plant photoreceptors.

One team from Russia produced transgenic tobacco plants overexpressing all four genes and studied the dynamics and distribution of luminescence, which mirrored phenylpropanoid metabolism variations, and its response to external signals. Another group from the USA took the next logical step and used the same fungal toolkit as an auto-luminescent quantitative reporter to study specific gene expression. Transient tests show that the approach works in diverse plant species. The luciferase gene is then driven by circadianand ABA-regulated promoters. The luminescence dynamics follows the expected patterns, detectable with a cheap setup.

The novel approach described in these two studies is still in the proof-of-concept stage. Optimization is necessary, particularly the recycling of the oxidized luciferin to avoid depletion of a substrate needed both for light emission and for the plant metabolism. But there is no question that having autonomous bioluminescent plants in the near future will be a plus for research that needs easily trackable biosensors and gene markers. Science fiction is happening, now.

Guillaume Tena

Published online: 11 November 2019

https://doi.org/10.1038/s41477-019-0558-x 